\title{
Erratum to: Black leaf spot of Japanese persimmon (Diospyros kaki), a new disease caused by Adisciso kaki sp. nov.
}

Jun Yamamoto $\cdot$ Kazuaki Tanaka •

Nobuaki Ohtaka $\cdot$ Toyozo Sato

Published online: 26 April 2012

(C) The Phytopathological Society of Japan and Springer 2012

Erratum to: J Gen Plant Pathol (2012) 78:99-105

DOI 10.1007/s10327-012-0367-9

Two errors appeared in the article. The GenBank accessions AB883952-AB883955 in left column of p. 100 should be AB683952-AB683955.

The online version of the original article can be found under doi:10.1007/s10327-012-0367-9.

J. Yamamoto

Shimane Agricultural Technology Center, Izumo,

Shimane 693-0035, Japan

e-mail: yamamoto-jun@pref.shimane.lg.jp

K. Tanaka

Faculty of Agriculture and Life Sciences, Hirosaki University,

Bunkyo-cho, Hiroskaki, Aomori 036-8561, Japan

N. Ohtaka

Life Science Research Institute, Kumiai Chemical Industry Co.,

Ltd, Kikugawa, Shizuoka 439-0031, Japan

T. Sato $(\bowtie)$

National Institute of Agrobiological Sciences,

Tsukuba, Ibaraki 305-8602, Japan

e-mail: s1043@affrc.go.jp 